\title{
Glioblastoma detection and therapy monitoring by microvesicle release
}

A micro nuclear magnetic resonance system ( $\mu \mathrm{NMR})$ to quantify microvesicle release from glioblastoma multiforme (GBM) and profile their protein expression has been developed by researchers at Massachusetts General Hospital, Boston, USA. According to Hakho Lee, one of the study leaders, "exosome numbers and protein expression can be used to assess tumour progression and to monitor treatment efficacy in GBM."

\section{4 ...GBM microvesicles can be distinguished from non-GBM microvesicles... 77}

Tumours in the CNS are not commonly associated with detectable soluble biomarkers; however, GBM is known to shed large numbers of microvesicles into the circulation. These microvesicles consist of exosomes, membrane particles, apoptotic vesicles and exosome-like vesicles that differ in size, and in expression of cell surface proteins.

A research team led by Lee and Ralph Weissleder quantified microvesicle numbers in a human GBM cell line, using magnetic nanoparticles targeted to microvesicle surface proteins to enhance NMR signal detection. The investigators used microvesicle size and CD63 expression to profile GBM microvesicles. By normalizing protein expression levels to the NMR signal, microvesicle numbers can be quantified in multicellular environments.

"Our goal was to develop a reliable assay to detect exosomes and to distinguish exosomes originating from gliomas from all other exosomes in the body," explains Lee. On the basis of their distinct molecular signature, including endothelial growth factor receptor (EGFR), EGFRvII, PDPN and IDH1 expression, GBM microvesicles could be distinguished from non-GBM microvesicles in blood samples.

Lee and colleagues also investigated the potential of the $\mu$ NMR system and microvesicle profiling to monitor GBM responses to drug therapy. The authors created a tumour progression index to monitor tumour size, which was based on a drug response index that reflected changes in the relative levels of GBM microvesicle numbers and surface protein expression. When tested in patients, the tumour progression index predicted treatment outcomes for responders and nonresponders in line with prognosis by an neuro-oncologist.

This innovative approach could potentially provide new avenues for cancer diagnostics. "We envisage that disease specific microvesicles will emerge as an important class of biomarker for early disease detection and monitoring," Lee explains. The high sensitivity and micro

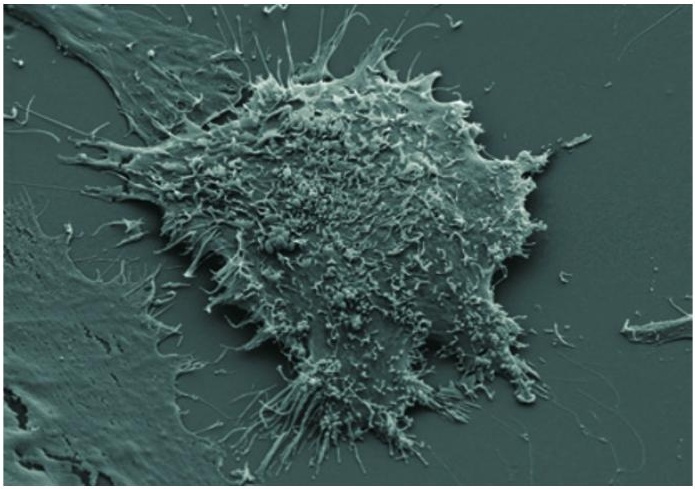

Scanning electron microscopy image of exosomes at the cell surface. Image courtesy of L. Hakho and R. Weissleder.

scale of this technology suggest that it could have applications in a clinical setting: as Lee suggests, "the $\mu$ NMR system is portable and easy to use, which makes it ideal for point-of-care diagnostics."

The researchers hope to develop new devices to allow microvesicle detection directly from whole blood, and are currently extending their research to include other types of tumours and microvesicle markers such as mRNAs and microRNAs.

\section{Ellen Bible}

Original article Shao, H. et al. Protein typing of circulating microvesicles allows real-time monitoring of glioblastoma therapy. Nat. Med. doi:10.1038/nm.2994 